\title{
Update in Contrast-Enhanced Ultrasound
}

\author{
Steffen Beckmann ${ }^{\text {a }}$ Jörg H. Simanowski ${ }^{\text {b }}$ \\ apractices Lachem, Hessisch-Oldendorf, Germany; ${ }^{b}$ Clinic for General, Visceral, Vascular and Obesity Surgery and \\ Interdisciplinary Emergency Center of the Nordstadt Clinic of the Hannover Region Clinic, Hannover, Germany
}

\section{Keywords}

Ultrasound · Ultrasonography · Echo contrast sonography . Abdomen $\cdot$ Imaging

\begin{abstract}
Background: The aim of modern medicine is to safely classify diseases for successful therapy without invasive measures. Sonography, computed tomography (CT), and magnetic resonance imaging (MRI) are potent imaging techniques. However, without contrast medium, the informative value of the 3 native methods is limited. The advantages of sonography are: no radiation exposure or previously known physically harmful interactions with tissue, proportionate disappearance of a contrast agent risk, no (probably irreversible) contrast agent deposits, and no risk of renal insufficiency. But, is that enough to compete with of even exceed CT and MRI? Summary: In this review, the state of the art of contrast-enhanced ultrasound (CEUS) in the abdominal cavity is presented. The remarkable diagnostic possibilities can unfortunately only be demonstrated here in a small number of impressive, typical case studies underpinned by the literature, so that, from one's own perspective, the full spectrum of CEUS can be used by oneself or initiated. Within the limits of physics, the real-time dynamics of CEUS enable conclusions to be drawn, so that with the current technology, sonography, including expansion by contrast, can be considered superior to other imaging methods. It is not uncommon for CEUS to have the value of a control and reference method. Key Messages: Sonography very often enables reliable diagnostics. The introduction of a contrast agent in sonography has led to a quantum leap similar to that of other imaging techniques. Already natively, the real-time representation of dynamic events leads to a certain superiority, i.e.,
\end{abstract}

complete observation of the inflow and outflow phases of the contrast medium and the resulting diagnostic; tissuespecific differentiation options provide a unique selling point. Further advantages of the first-choice imaging diagnostic method are: a lack of radiation exposure, repeatability of the examination at any time, local independence, a negligible allergy rate compared to the contrast agents of other methods, and a lack of kidney and thyroid exposure or excluded deposits.

(c) 2020 S. Karger AG, Basel

\section{Introduction}

Echo contrast sonography (contrast-enhanced ultrasound; CEUS) has developed into a highly specialized area of medical diagnostics. As with computed tomography (CT), the introduction of contrast media in sonography revolutionized diagnostic precision. In the hands of experts on ultrasound devices with CEUS-capable hardware and software, CEUS is considered superior to other imaging methods, which often pollute many organ systems with radiation. The unique selling point for imaging methods is real-time representation of the inflow and outflow behavior of the contrast medium in the tissue. This is often characteristic and comes close to noninvasive, histological differentiation. The contrast medium used is a purely intravascular agent without interaction with the thyroid or kidneys. It clearly has clearly lowest contrast agent allergy rate [1]. This article is intended for all those who want to use the value of this technique in the context of their own diagnostic strategies through the targeted initiation of a CEUS examination. 


\section{B-Scan Sonography: The Basis of CEUS}

The examiner generates the groundbreaking differential diagnostic considerations from the grayscale image. Contour, cut surface, internal structure, the relation to the surrounding tissue, infiltrative, and cross-organ or repressing growth can be seen. Signs of inflammation and size are key parameters.

In the liver, the courses and branches of the supplying (portal veins and arteries) and draining (liver veins) vessels as well as the bile duct can be traced as far as possible. Their nature influences the echogenicity of the liver parenchyma just as, conversely, parenchymatous diseases affect the lumens of the above-mentioned vessels.

Once these relationships have been worked out in the fundamental gray value sonography, the administration of a signal amplifier can be used to strengthen or exclude considerations resulting from the gray value image. In terms of its informative value and persuasiveness, CEUS is therefore dependent on the quality of the gray value sonography and it is not a diagnostic agent per se.

The liver is the only abdominal organ with a dual blood supply. While the portal vein supplies the crucial amount blood required for the trophic process of the liver, the arteries primarily supply the biliary tract. Microscopic connections between portal branches and arteries are inherent in the liver and play a role in vascular pathologies and parenchymatous diseases. Additional vessels that supply the liver transcapsularly, paraportally, or paraligamentarily can cause differences in echogenicity and temporary contrast phenomena.

Pathological, parenchymatous, or focal processes deviate from the normal expected blood flow pattern due to changed angioarchitecture, vascularization, and thus perfusion. Non-liver tissue and liver tumors show a changed vascularization pattern compared to the surrounding liver parenchyma.

The blood flow velocities, volumes, and pressures listed in Table 1 apply to the healthy liver of a patient who does not have heart failure, the knowledge of which is essential for the assessment of pathological vascularization phenomena (Table 1).

In addition to the highest spatial resolution of the imaging, the advantages of CEUS lie in the ability to record the smallest vessels and in dynamic recording of blood circulation properties. In order to simulate real-time conditions in CT, several short spirals would have to be run, which massively increases the radiation exposure. The spatial resolution of the ultrasound would still be better.

Many studies have shown that CEUS is not inferior to $\mathrm{CT}$ or magnetic resonance imaging (MRI) in its informative value in various areas of application [2].
Table 1. Duplex sonographic parameters in liver vessels and flooding time in the liver after peripheral venous administration in healthy people [5]

\begin{tabular}{lcccl}
\hline & $\begin{array}{l}\text { Pressure, } \\
\mathrm{mm} \mathrm{Hg}\end{array}$ & $\begin{array}{l}\text { Flow, } \\
\mathrm{cm} / \mathrm{s}\end{array}$ & $\begin{array}{l}\text { Volume, } \\
\mathrm{mL} / \mathrm{min}\end{array}$ & $\begin{array}{l}\text { Time to arrival of } \\
\text { the contrast agent } \\
\text { in the vessel after } \\
\text { injection, } \mathrm{s}\end{array}$ \\
\hline $\begin{array}{l}\text { Liver artery } \\
\text { Portal vein }\end{array}$ & 100 & 100 & 200 & $\begin{array}{l}\text { Up to } 15 \\
15-30 \\
\text { Liver vein }\end{array}$ \\
\hline
\end{tabular}

Tables on contrast phenomena in CEUS are limited in their statements because they only phrase the characteristics of microvascularization schematically and do not take into account the change in the blood supply of a focal liver lesion at different times of its existence.

\section{How Does CEUS Work?}

Ultrasound diagnostics is a pulse-echo method and visualizes what is reflected from the tissue back to the transducer after the ultrasound pulse has been emitted. This is an important difference compared to the transmission of $\mathrm{X}$-ray and MR procedures.

The differences in the brightness of the grayscale image are based on the different acoustic impedance of the tissue types; the greater the difference in impedance is, the stronger the reflection is. Blood (mainly erythrocytes) has little backscatter and is therefore "black" in the gray value image. Microbubbles are used to increase the backscattering of the blood.

Second-generation contrast media consist of waterinsoluble gas, i.e., sulfur hexafluoride, surrounded by a phospholipid monolayer membrane. Ninety percent of the microbubbles have a diameter $<5 \mu \mathrm{m}$ and thus allow multiple lung passages before they are exhaled at the gasgas interface of the lung alveoli after $20-30 \mathrm{~min}$ [3]. These microbubbles increase the backscatter by a factor of about 1,000 due to the impedance jump generated at the bubble membrane as an interface and can be viewed physically as an elastic balloon that vibrates depending on the sound intensity.

At the beginning of contrast ultrasound, the bubble destruction by high acoustic energy (power Doppler) is used to draw conclusions about the vascular density of a tissue (Fig. 1). Uncolored areas represent tissue of a poor vessel density [4].

Today, a low mechanical index allows moderate oscillation of the bubbles so that they do not burst too quickly and persist for a long time and so the angioarchitecture can be assessed. 
Fig. 1. High mechanical index $(>1)$ in power Doppler mode leads to disruption of the microbubbles, indicating highly vascularized tissue (currently known as replenishment or flash mode). Not-highlighted areas represent a low vascularity.

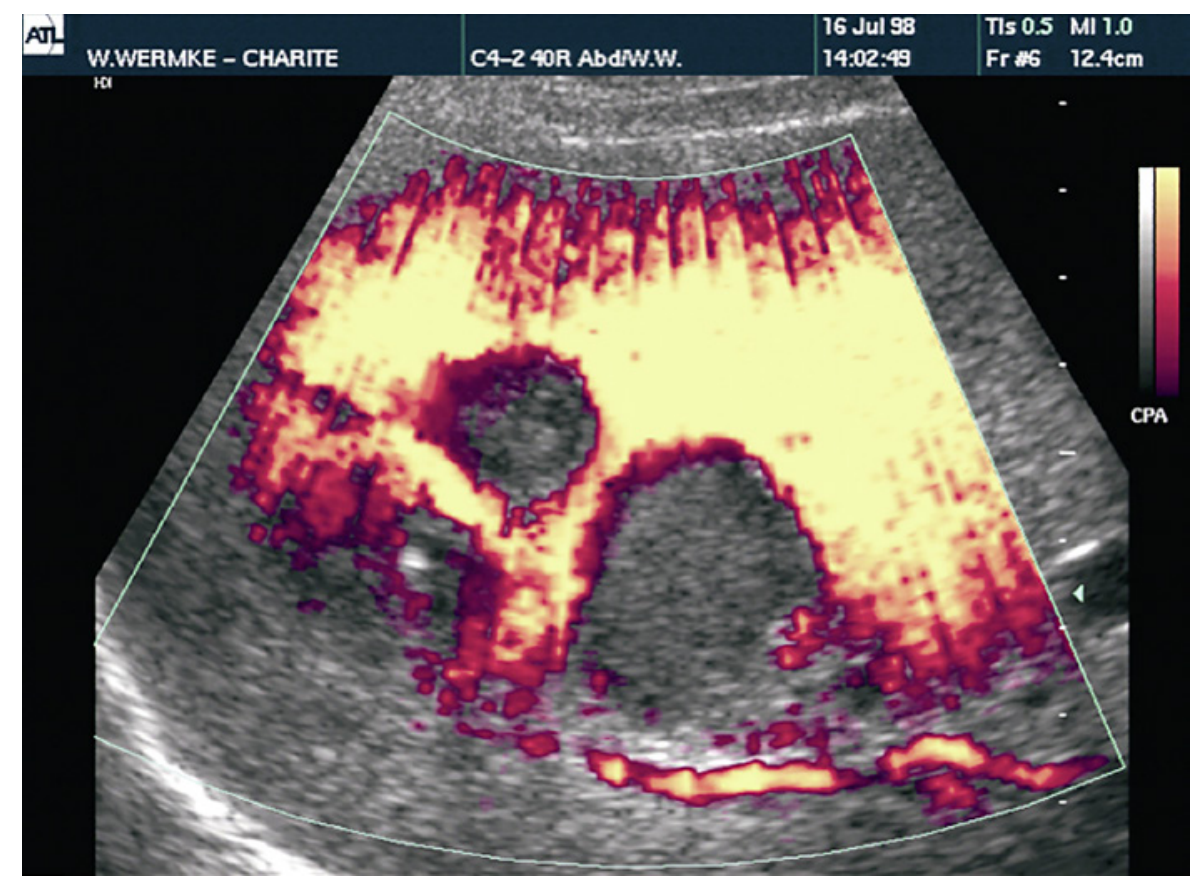

The contrast ultrasound image is an image of selected frequencies; bubbles excited by a suitable frequency (1.7 $\mathrm{MHz}$ or integer multiples) also vibrate at harmonic frequencies (harmonics) because the compression and decompression of the bubbles generate nonlinear vibrations at these frequencies. These so-called harmonics can be selectively represented by the ultrasound device and highlighted in color. The result is a pure contrast image (contrast harmonic imaging) comparable to digital subtraction angiography. In contrast, the tissue only emits linear vibrations in this frequency range. The linear backscattering of the tissue is usually eliminated by 2 pulses on 1 image line with a $180^{\circ}$ phase shift canceling out the linear backscattering while the nonlinear signals of the microbubbles are retained - harmonic imaging. One ampoule of SonoVue ${ }^{\circledR}$ contains approximately $1,000,000,000$ microbubbles ( $10^{8}$ or 100 million) after dissolution in $4.8 \mathrm{~mL}$ of isotonic saline. Microbubbles can be detected in vessels with diameters in the range of $0.1 \mathrm{~mm}$.

The representation of blood circulation with ultrasound contrast medium is based on the vibration properties of the microbubbles. Depending on the applied transmission power (acoustic pressure), the microbubbles vibrate with increasing acoustic pressure with increasing nonlinearity - the bubble diameter can no longer follow the acoustic pressure proportionally. This creates the harmonics - the harmonic frequencies that are detected and processed for imaging the blood circulation without a disruptive tissue display.

\section{Focal Liver Lesions}

Angiomas are mesenchymal liver tumors without a risk of malignancy. They are common incidental findings in sonography, but they are also increasingly common in other nonliver slice examinations such as spine MRI and cardio CT/MRI. Since these modalities do not focus on the diagnostic of focal liver lesion, further assessment is frequently required. CEUS avoids large-scale device diagnostics and X-radiation by the CT.

Five types of angioarchitecture can be classified using CEUS [5]. A prerequisite for imaging type diagnosis is always an adequately preserved angioarchitecture. Since the vessels within the tumor degenerate over time, hemangiomas have a wide range of appearances on gray scale imaging. Their morphology is also subject to change of the echogenicity of the surrounding liver parenchyma (online suppl. Fig. 1; for all online suppl. material, see www.karger.com/doi/10.1159/000511352). On grayscale imaging, angiomas cannot generally be differentiated reliably from malignant tumors or metastases (Fig. 2).

Focal nodular hyperplasia (FNH) is a congenital vascular malformation. It is less common than angiomas and more common in females. Multifocal FNH or coexistence with hemangiomas is not unusual. A CEUS mark is early intranodal enhancement by the supplying artery (or arteries). The steal phenomenon is the preferred intranodal microbubble concentration at the expense of that of the parenchyma in the arterial phase; it is typical for FNH. Concentric arterial branching in type $1 \mathrm{a}$ and eccentric arterial branching in type $1 \mathrm{~b}$ are subdivided. The classic 


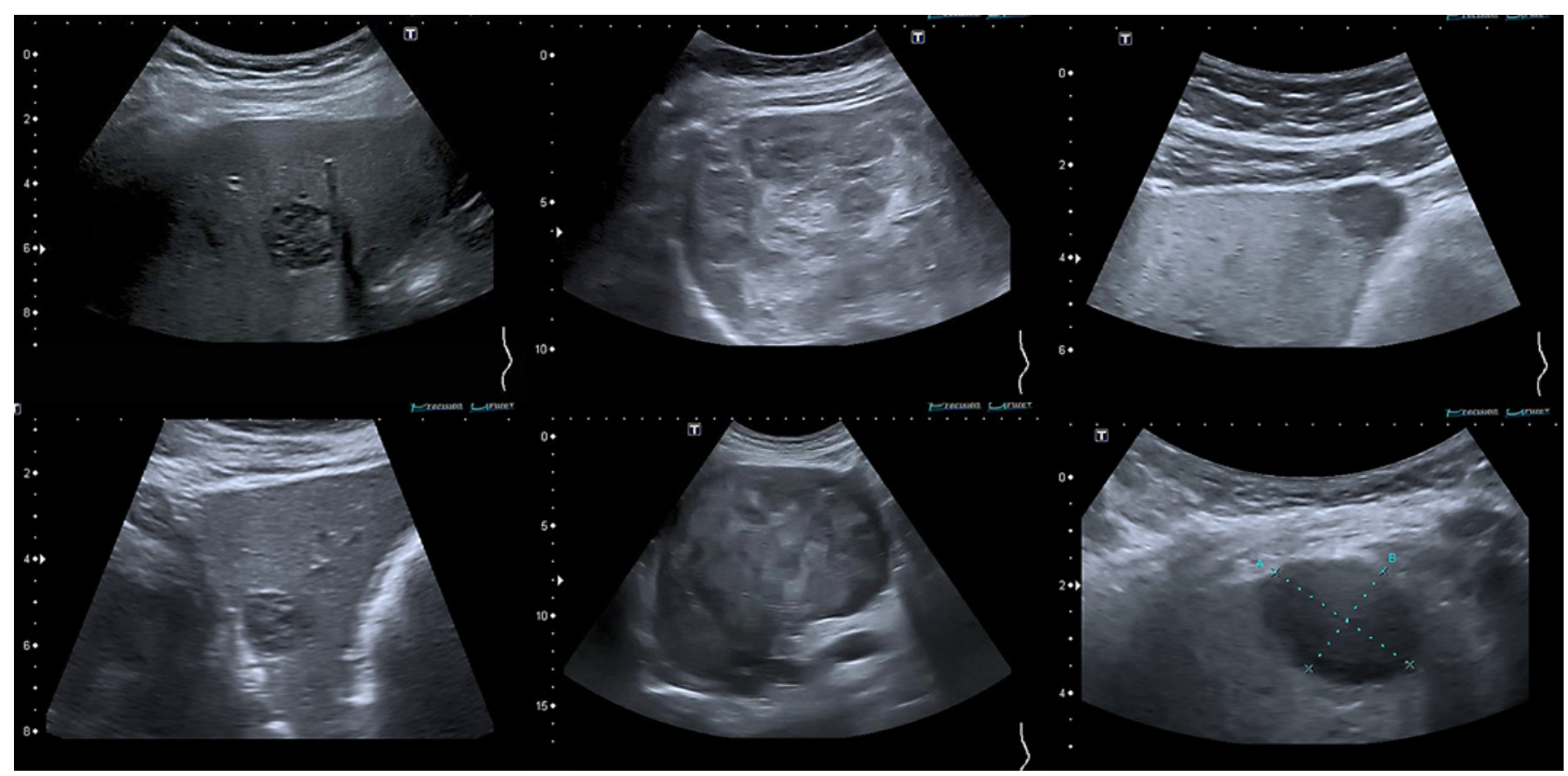

Fig. 2. Left: a metastasis of uveal melanoma looks similar to the angioma. Middle: the large angioma and the HCC cannot be distinguished in a gray scale image. Right: type 3 angioma and the metastasis of adenocarcinoma of the pancreas are indistinguishable without CEUS.

scar occurs in about $80 \%$ of cases. The direction of spread of the contrast agent is centrifugal and creates a wheel spoke pattern. FNH has arteriosystemic fistulas, which shift the blood from the tumor into adjacent veins. Depending of the amount of blood being drained, the vein may be dilated. On CEUS microbubbles appear in the draining vein seconds after reaching the tumor through its supplying artery (Fig. 3).

Histologically, FNH shows bile duct proliferation in which the liver-specific contrast medium of MRI examinations can be detected, allowing FNH to be distinguished from liver adenomas in case CEUS might not be conclusive.

\section{Malignant Liver Tumors}

Hepatocellular carcinomas (HCC) occur in cirrhosis of the liver and in the undeveloped liver and are less common than metastases. Focal lesion cirrhosis is suspicious for HCC until clarified. The diagnostic value of the CEUS compared to CT and MRI has been widely investigated [6]. In the cirrhotic liver, the development of a dysplastic node to HCC is the result of dedifferentiation. The changing angioarchitecture can be excellently represented by CEUS. Vessel-rich nodes with capsular vessels and variable caliber intranodal pulling tumor arteries, undirected branches and blindly ending tumor arteries are the hallmarks of CEUS (online suppl. Fig. 2). Decreasing portal perfusion is the result of neoangiogenesis and diminishes as it progresses. CEUS is an established and reliable method for the diagnosis of HCC and part of the guidelines [7]. CEUS is used for the detection of vital tumor residual tissue after radio frequency ablation (RFA) because of its capacity for visualization of tiny blood vessels.

Cholangiocarcinoma (CCC) is the second most common malignant liver tumor, with an increasing prevalence [8]. Risk factors include chronic inflammation of the bile ducts such as PSC, HCV infection, parasitic diseases (echinococcosis), and bile duct malformations such as Caroli disease. Intrahepatic, peripheral CCC is a connective tissue-rich tumor, lobulated and often polycyclic, with a lower echogenicity than that of often fatty liver parenchyma. Unimpeded transtumor vascular courses are seen frequently. Microsatellites can be found nearby. Calcification in the tumor may occur. The liver capsule can be retracted by fibrotization (umbilication). Pronounced arterioportal fistulas repealing the margin of the carcinoma immediately in the arteriel phase and a rapid intranodal decrease in the microbubble concentration are characteristic in CEUS (online suppl. Fig. 3).

Compression of adjacent cholangia results in biliary fibrosis of the affected segment. As a consequence, arterial perfusion is increased due to poor portal perfusion in this area. Thus, detection of CCC based on the expected low portal perfusion of the tumor in the portal phase can be difficult because of reduced differences in contrast between the tumor and the parenchyma. 


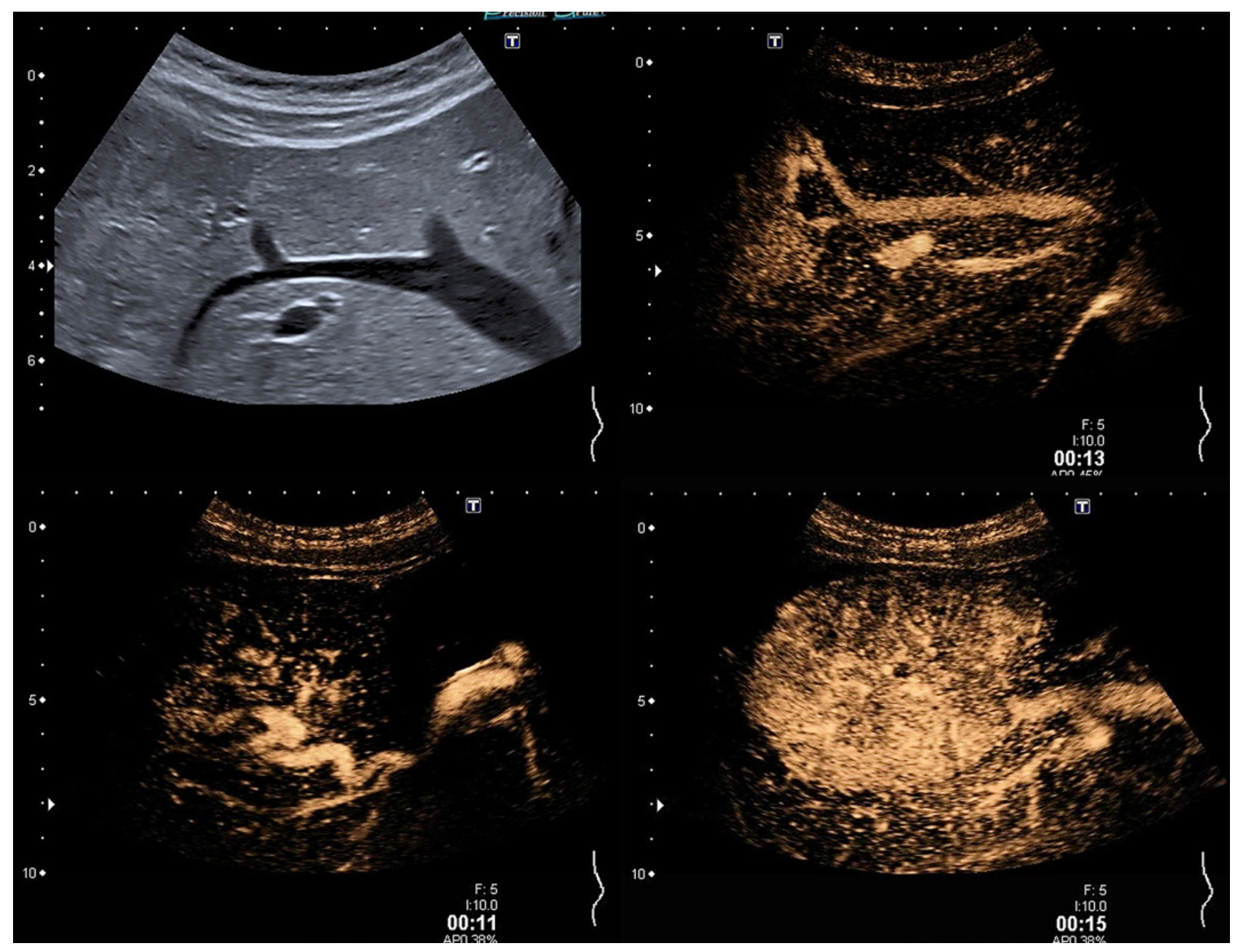

Fig. 3. FNH type 1a. Upper left: the middle hepatic vein seems dilated. The liver parenchyma left of the vein is inhomogeneous. Upper right: $13 \mathrm{~s}$ after the administration of contrast agent the dilated hepatic vein is filled with microbubbles. The right hepatic vein and the parenchyma show no bubbles. Lower left: feeding vessel (artery). Lower right: FNH with the typical scar.

\section{Liver Metastases}

Liver nodules, with or without a tumor history, especially if they are thought to be diagnosed for the first time, are suspicious for a nonhepatic origin. Their gray scale image is inconsistent and can often resemble benign liver nodes. The foreign tissue has a nontriadal, i.e., unpaired, angioarchitecture. For this reason, metastases in the phase of the maximum microbubble inflow into the liver, i.e., the portal phase, are noticeable due to a lower bubble concentration compared to the liver parenchyma (the so-called "washout phenomenon"). Their vascular supply can initially make the metastasis appear hypervascularized. Tumors with typically hypervascularized filiae include malignant melanoma, breast cancer, and neuroendocrine tumors. If the liver is interspersed with nodes, the diagnosis of metastatic liver is easy with "washed out" nodes with any imaging method. If it is a question of singular masses with cystic or necrotic parts or of settlements next to benign nodes, the distinction from metastasis is more difficult (online suppl. Fig. 4). The advantages of CEUS are spatial resolution, dynamics of the blood flow, and fractional bolus administration of the contrast medium for selective display of nodes.

\section{Abscesses and Intracavitary CEUS}

The diagnosis by clinic, laboratory analysis, and imaging has proven to be variable for each of these criteria and they do not always coexist with each other. Just as there are uncertainties in clinical and laboratory parameters, the fundamental B-scan sonography is not always 


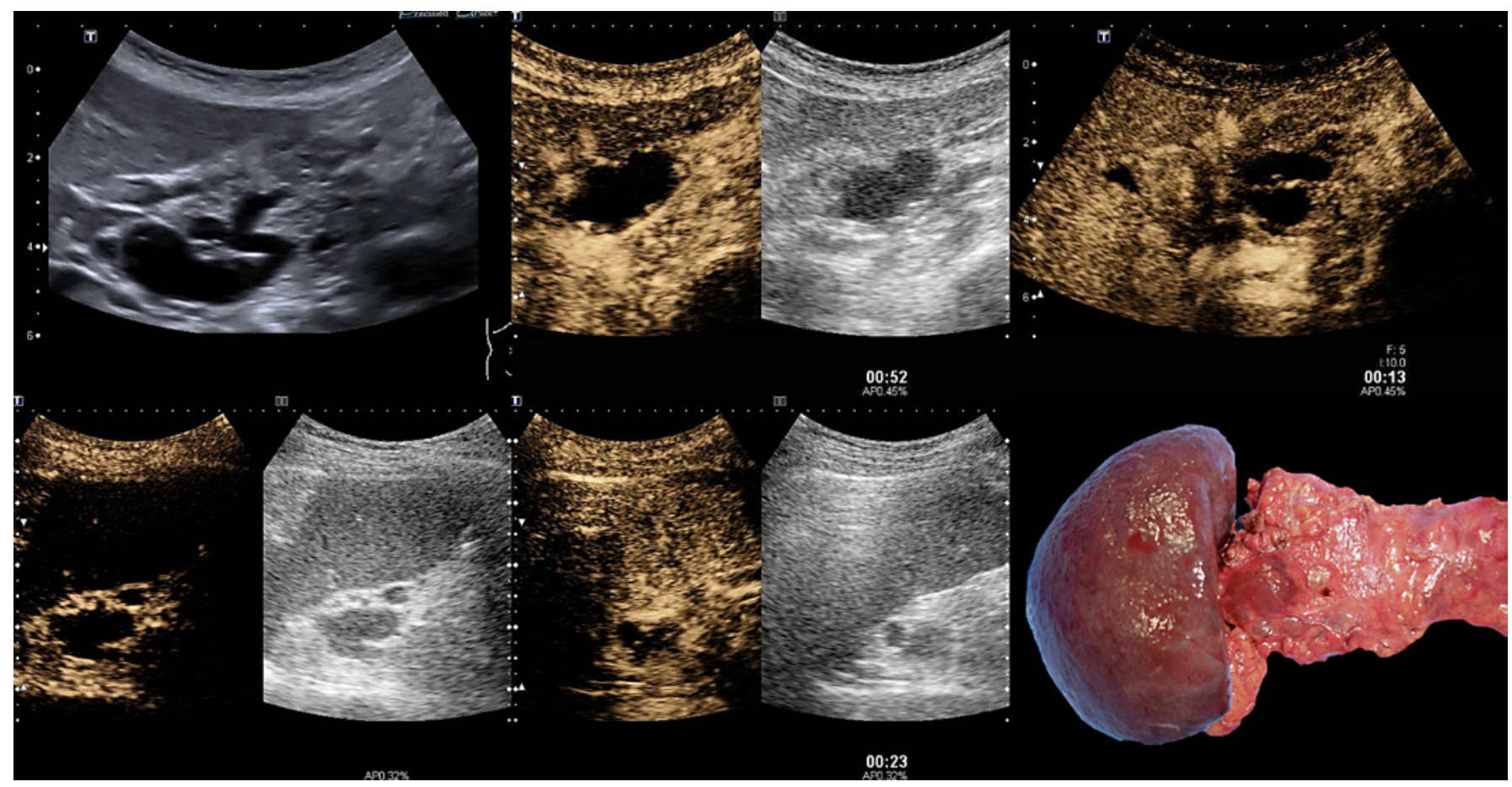

Fig. 4. Top: cystic lesion on the head of the pancreas, spreading to the duct of Wirsung. Noduli and septa are shown. CEUS shows contrast-enhanced nodules and septa. Bottom: cystic lesion at the tail of the pancreas (probably nonvital). CEUS shows thin septa and nodules rising from the wall of the cystic lesion. The resected tissue shows the intraductal papillary mucinous neoplasm in the head and tail of the pancreas.

clear, except for the stage of the encapsulated abscess. For example, abscesses caused by Klebsiella pneumoniae often appear like solid tumors [9]. Depending on the stage of maturation of a liver abscess, 4 groups can be distinguished using the CEUS pattern [10]. CEUS provides valuable information for both intravenous and intracavitary applications as part of the intervention [11]. For the microbiological examination in stage I, the sonographer can only distinguish avital, necrotic tissue from ischemic tissue using CEUS, which is why CEUS is recommended before the material is obtained. CEUS can better measure the dimension of an abscess, follow the healing process, and significantly increase the detection rate of smaller abscesses compared to fundamental images [12] (online suppl. Fig. 5). Intracavitary CEUS provides information about the correct position of the drain and communication of the necrosis cavity with vascular systems, in the liver with the biliary tract (online suppl. Fig. 6) [13-15] or with liver veins, and it is also used for the detection of vesicourethral reflux [16]. In general, fistulas between neighboring structures and anastomotic insufficiency can be visualized with CEUS [17-19].

\section{Pancreas}

Cystic pancreatic lesions, like other masses in the abdomen, are often incidental and rarely the result of a targeted search for specific symptoms. B-scan sonography is established in the detection of a pancreatic mass and can distinguish solid structures from cystic ones. CEUS provides pioneering information on the differential diagnostic classification and on how to proceed. In the case of cystic structures, nodules, very fine perfused septa, and wall perfusion can be worked out better than in CT (Fig. 4). The distinction between mucinous and serous neoplasia is not inferior to that achieved with MRI [20]. Intraductal papillary mucinous neoplasm is less common than the aforementioned cystic pancreatic lesions. The connection to the pancreatic duct is required for diagnosis. A passage width of 5-9 $\mathrm{mm}$, a size $>3 \mathrm{~cm}$, and pancreatitis followed by obstruction are warning signals for which degeneration cannot be ruled out [21-23].

Solid pancreatic nodes can be better differentiated using CEUS. In blood flow assessment in CEUS, a distinction is made between an arterial phase (10 s), a parenchymatous phase (10-20 s), a venous phase ( $>60 \mathrm{~s})$, and a late phase ( $>120 \mathrm{~s}$ ). While $90 \%$ of ductal adenocarcinomas are noticeable as a result of desmoplastic stroma and fibrosis due to a lower vascular density and thus less vascularized 


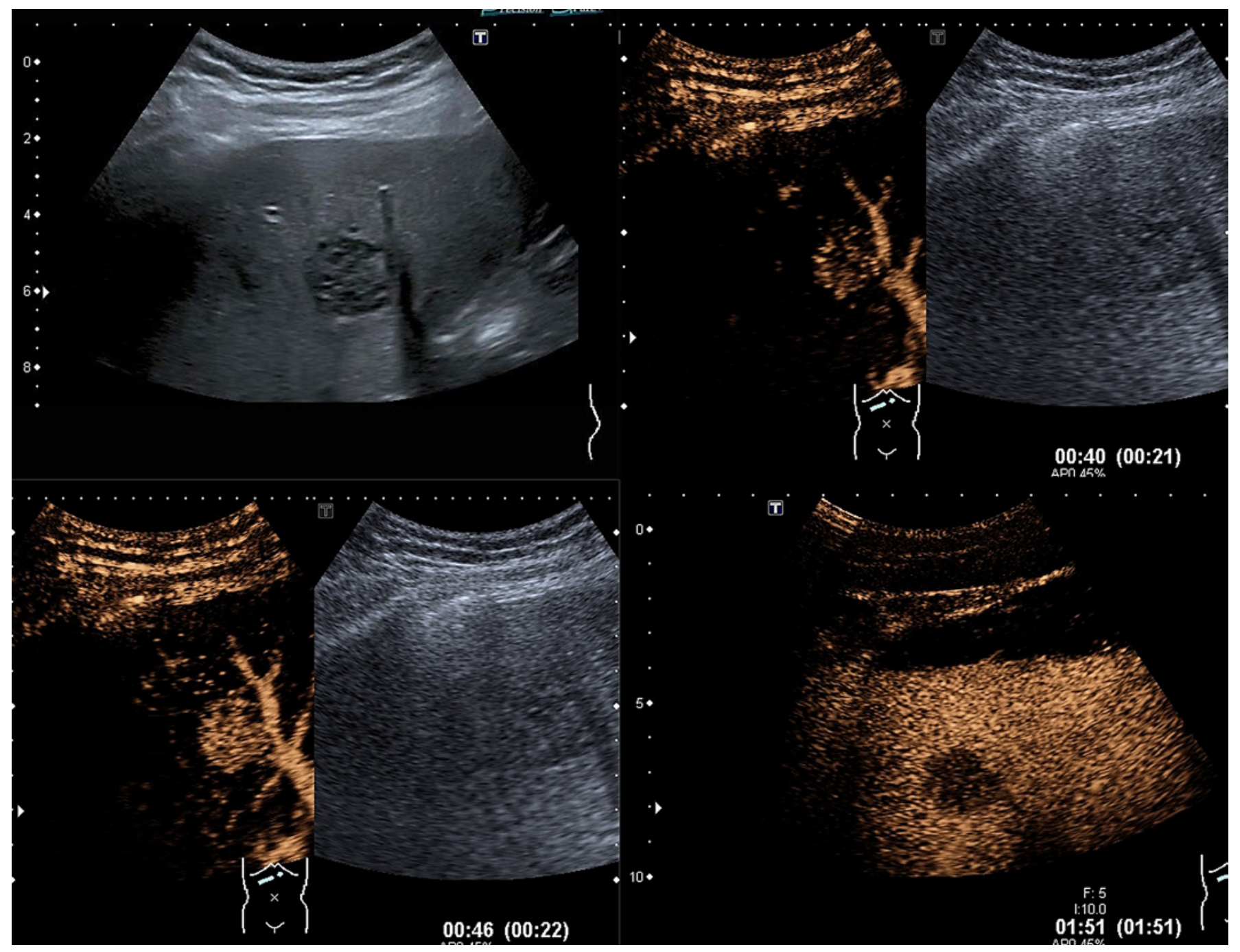

Fig. 5. Follow-up after therapy of uveal carcinoma. On a gray scale image the liver mass could be mistaken for an angioma. CEUS shows a high inflow of microbubbles into the tumor. The vascular pattern does not match the presumed angioarchitecture. About $60 \mathrm{~s}$ after administration of the contrast agent the bubble concentration of the tumor decreases. Metastases of melanomas are typically highly vascularized, as seen here. Lesion was not detected in CT und misinterpreted in MRI.

compared to the surrounding pancreatic tissue (online suppl. Fig. 7), neuroendocrine tumors are vascularized and hypervascularized compared to the parenchyma of the pancreas $[24,25]$. If B-scan and CEUS speak for adenocarcinoma, fanning out of the liver, which can be carried out at the same time, helps to find distant metastases. Adjacent lymph nodes and the relationship to the visceral aortic branches provide information about the therapeutic approach. In the palliative approach, these measuring points provide orientation when assessing the response or progress.

Focal pancreatitis cannot be clearly distinguished from adenocarcinoma in the B-scan and, if it is appropriately localized, it may cause the biliary obstruction described for both entities (intrahepatic "double shotgun sign"). CEUS can help to differentiate poorly vascularized ade- nocarcinoma from focal inflammation based on the vascular density, in which the contrast medium floods somewhat more slowly due to the concomitant vasculitis and persists significantly longer than in carcinoma [26].

\section{Intestine}

The inflammatory activity of the intestinal wall is described by the Doppler method in Crohn disease with the Limberg classification. By quantifying the contrast medium enhancement in the affected wall layers, CEUS offers a good correlation with the Crohn activity index [27]. The response to a therapy can also be assessed by displaying intestinal wall enhancement $[28,29]$. There are specific contrast ultrasound features for Crohn disease: the 
inflows that supply the intestine, the vasa recta, appear to spread out of the mesenteric root (crest mark) due to fibrolipoma-like inflammation of the fatty tissue. The zone sign describes the increased vascularization in the tunica mucosa and subserosa in favor of the less perfused muscularis [30]. If there are fistulas or abscesses, the advantages of intracavitary CEUS are better fistula and abscess visualization as well as visual control in the intervention.

\section{Other Tumors of the Gastrointestinal Tract}

These are usually discovered accidentally in sonography. CEUS can represent vascularization. Subepithelial tumors, NET, and GIST (online suppl. Fig. 8) are hypervascularized but may have necrotic areas, especially in large tumors. If a biopsy is planned, CEUS can differentiate between necrotic and vital tissue and reduce the risk of misguided biopsy. Distinction from leiomyoma is possible using CEUS [31].

\section{Oncological Issues}

In addition to the direct question of malignancy in the case of unclear masses, follow-up monitoring under treatment of a tumor is an important control element in oncology. The response behavior and therapeutic effect are assessed for many entities via imaging. Tumor follow-up protocols differ in their imaging recommendations. The often practiced routine of quarterly CT examinations, often paired with a subsequent liver MRI, is worth considering.

For colorectal carcinoma, CEUS has been established for the detection of liver metastases [32]. B-scan sonography in the detection of small metastases is limited by the scattering of the sound waves, particularly in the case of therapy-related fatty liver formation. The response to a therapy attacking the tumor angiogenesis by CEUS can be recognized after a short time; the decreasing vascular density and metastatic vascularization become clear in CEUS and correlate with the progression-free survival [33-36]. Ultrasound is part of the aftercare for choroidal melanoma. In this context, unclear liver foci can be excellently clarified with CEUS and, in the case of metastasis detection, underline the indication for PET-CT, which is often initially refused by the health insurance (Fig. 5; online suppl. Fig. 9-12).

\section{Postoperative Issues}

There are no established regimes for postoperative procedures after interventions in the abdomen due to the variety of potential questions. The focus is usually on wound healing and the clinical course. There are 2 reasons for ultrasound as the first choice for imaging in the postoperative course; first, unlike in radiology, the user of the imaging is always a clinical examiner, and second, the ultrasound can be repeated an unlimited number of times if the findings require control or are unclear (online suppl. Fig. 13).

In addition, it can also be checked in this context, e.g., in case of nonconclusive histological findings, whether the objective of an intervention has been achieved.

\section{Intraoperative CEUS}

Already at the end of the 1980s, the overlay-free, intraoperative, intracorporeal, then still pure, high-frequency grayscale sonography with inspection and palpation had been proven to be clearly superior to preoperative use and CT with contrast media. In about $20 \%$ of cases, additional findings in the liver led to a changed surgical procedure [summary in 37]. With the introduction of ultrasound contrast agents, as with contrast CT, there was a leap in quality so that CEUS became the method of choice for validating parenchymal masses. The advantage of intraoperative, intracorporeal B-scan ultrasound (ICUS) was gone, especially since the hardware and software for bubble detection were not compared to the high-frequency transducers that can be used intraoperatively. The same low-frequency transducers had to be used for ICUS as for the transcutaneous sound, with only slightly more sensitive detection. Transducer frequencies up to $12 \mathrm{MHz}$ and CEUS with up to $7.5 \mathrm{MHz}$ are currently used. The old lead has been restored, so that results can again be collected in at least $20 \%$ of cases, which leads to a change in the preoperatively planned procedure. Intraoperatively it is not primarily about type diagnosis with CEUS but about the highly sensitive first discovery of a tumor. One of the focal points is now direct therapy control of thermoablative processes by CEUS, independently of whether a sufficient safety margin has been reached or whether further measures have to be taken (Fig. 6).

\section{CEUS in Trauma}

Free fluid is the highly sensitive indicator of a relevant trauma [38]. The cause cannot always be immediately visualized with B-scan sonography. CEUS has established itself as a tool to assess the extent of injury to a parenchymatous organ. The homogeneous contrast of the regular blood flow to tissues or vessels compared to the failure of traumatically damaged tissues or vessels represents a oneglance diagnosis. In some cases, the ruptured vessel can be directly identified through the microbubble outlet. 


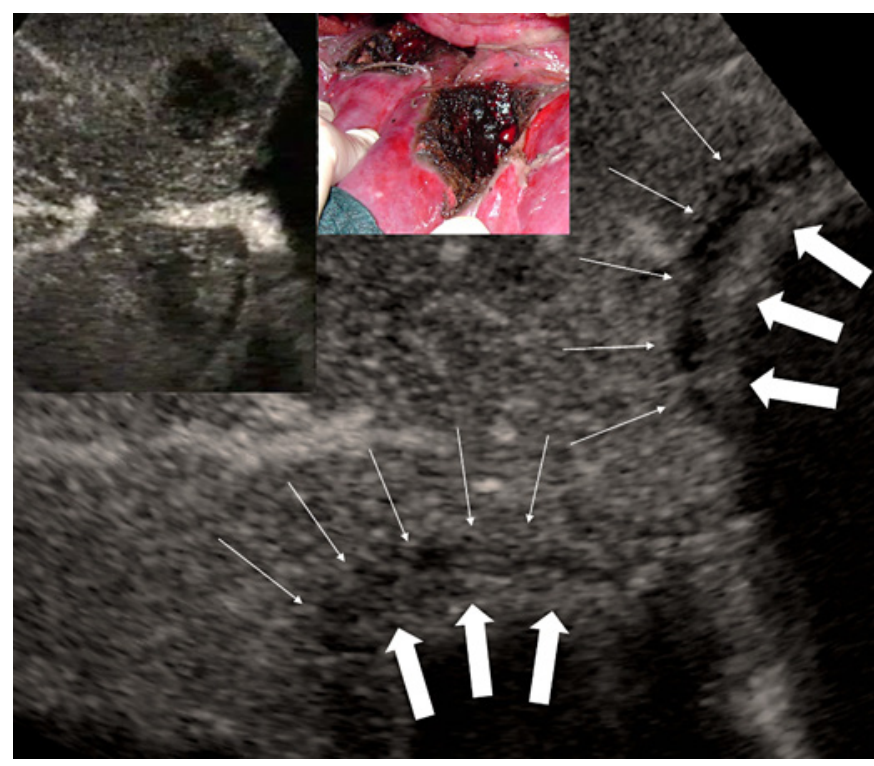

Fig. 6. Two metastases directly in the hepatic vein star. Intraoperative CEUS for determination of the thermoablative active zone in the tissue beyond the immediate, necrotized zone: wide arrows indicate vaporized necrosis, while narrow arrows indicate a destroyed, no longer perfused tissue margin (i.e., a sufficient safety margin, experience has shown that this void-free zone widens further within $24 \mathrm{~h}$ ).

\section{Discussion}

Signal-enhanced sonography with contrast medium (CEUS) is a proven instrument in a wide field of diagnostic imaging. Numerous studies have confirmed the benefits of CEUS and highlighted its value compared to CT and MRI. CEUS has left the position of an additive procedure for nonconclusive CT or MRI findings and represents a first-class diagnostic tool that can be followed by radiological sectional imaging procedures in the sense of staging with the appropriate indication.

Focal liver lesions can be finally clarified in CEUS. For the diagnosis of HCC, CEUS is an integral part of the guidelines. It is unclear why CT is still considered the gold standard for control after RFA, although the advantages of CEUS in carcinoma detection also come into play in control after RFA.

For small lesions in particular, CEUS is technically superior to CT and MRI in resolution [39]. The real-time procedure has advantages over the more static and prospectively oriented contrast agent examination by CT or MRI. Vascularization patterns of the HCC in the arterial, portal venous, and late phases have been described inconsistently in various studies and depend on the degree of dedifferentiation of the tumor [40]. In the crucial arterial phase of HCC perfusion, no meaningful interobserver variability was found [41]. In this respect, CEUS is ideally suited as a real-time method for detecting and clarify- ing tumors based on their individual angioarchitecture. HCC below $15 \mathrm{~mm}$ in size are more difficult to detect with MRI [42]. In principle, small-sized tumors can be avoided by CT and, similarly, by MRI as a result of the partial volume effect of the diagnosis. In late contrast declines, as in some G1 HCC and in small neuroendocrine filiae, CEUS is superior to CT in terms of detection.

Like all cross-sectional imaging methods, CEUS cannot prove the origin of metastatic nodes. The precision in the detection of metastases has been proven many times, especially for colorectal carcinoma. Hypervascularized metastases can arise from various tumors. Metastases of neuroendocrine tumors are prone to necrosis, as are some colonizations of colorectal carcinomas. The advantage of CT lies in a better overview, whereby the detection of small metastases, HCC, NET, or NEC, especially in the intestine, is better achieved using high-resolution methods or methods linked to metabolism.

Air distribution, sound windows, and penetration depth are often obstacles in sonography that cannot be influenced. The often described examiner dependency of ultrasound has 2 meanings. On the one hand, insufficient technical knowledge in sonication using microbubbles as well as a lack of experience and training in examination technology are limiting factors that apply not only to ultrasound. On the other hand, the method can deliver excellent and amazingly convincing images of pathological processes in the trained and practiced hand. Technical factors exist for both radiological and sonographic methods. Ultrasonic machines have device-specific software and are not identical in handling CEUS. Depending on the ultrasound machine, the quality of image reproduction can be changed via numerous settings and requires good knowledge of adjustment. In CT, the iodine concentration and bolus size and flow, as well as the time of image acquisition via fixed delays or ROI-triggered contrast agent concentration phases per se, should not be chosen in a lump sum manner in every patient and are therefore parameters that influence the examination quality.

Pancreatic space requirements are usually initially identified using gray-scale ultrasound. It is not always possible to differentiate between cystic and solid structures. The smaller the knot is, the more difficult it is to distinguish. Nodes smaller than $20 \mathrm{~mm}$ in size can escape CT or cannot be classified with sufficient accuracy [43].

CEUS is reliable in differentiating solid modules from cystic ones and shows septa and nodules of cystic tumors just as clearly as MRI. In differentiation within cystic neoplasia, CEUS is superior to CT and it is equivalent to MRI [44]. The most common solid mass of the pancreas is ductal adenocarcinoma, which is recognized with a high accuracy and can be distinguished very well from neuroendocrine neoplasia as the second most common solid neoplasia of the pancreas. 
Differential diagnostic difficulties arise for transabdominal CEUS in the small microcystic serous neoplasia, which, due to the microcystic structure, can simulate a hypervascularized tumor. The endosonographic representation as ECEUS can dissolve the angioarchitecture and is therefore helpful in delimitation of solid hypervascularized nodes [25].

Although sonography is not the method of choice for locating gastrointestinal tumors, ultrasound can do valuable work. Subtle intestinal ultrasonography often uncovers the primary in the jejunum when searching for primary liver metastases suspicious for NET. Hypervascularized tumors can be assigned to the common subepithelial tumors NET or GIST. The indication of their location serves as an important indication for the subsequent staging examination. If intestinal tumors are to be biopted as part of a histological backup, CEUS can define necrosis areas and improve the success rate of sampling.

\section{Conclusion}

With CEUS as a low-complication supplement to the excellent gray value sonography, the clinician is provided with a valuable diagnostic procedure for a wide range of indications, which provides immediate information for completing the diagnosis or for initiating further diagnostics or therapy steps. In coexistence with radiological sectional imaging, it has replaced its dominance in many areas. The lack of an overview and physical constraints generally limit ultrasound as the sole examination medi$\mathrm{um}$. Whenever it is necessary to demonstrate pathological processes in terms of image morphology, ultrasound is the first diagnostic tool and, in conjunction with CEUS, a first-class procedure.

\section{Statement of Ethics}

Since this is a review article, institutional approval and patient consent were not required.

\section{Conflict of Interest Statement}

S.B. has no conflict of interest to disclose. J.H.S. declares that he has received lecture fees from Mylan Healthcare $\mathrm{GmbH}$.

\section{Funding Sources}

The authors received no funding for this paper.

\section{Author Contributions}

S.B. wrote this paper. J.H.S. critically revised this work and added the paragraph on intraoperative sonography and CEUS in trauma.

\section{References}

1 Weskott HP. Contrast-enhanced ultrasound. 2nd ed. London: Uni-Med Science; 2013. p. 240.

2 Seitz K, Strobel D, Bernatik T, Blank W, Friedrich-Rust M, Herbay A, et al. ContrastEnhanced Ultrasound (CEUS) for the characterization of focal liver lesions - prospective comparison in clinical practice: CEUS vs. CT (DEGUM multicenter trial). Parts of this manuscript were presented at the Ultrasound Dreiländertreffen 2008, Davos. Ultraschall Med. 2009 Aug;30(4):383-9.

3 Greis C. Erratum zu: Technische Grundlagen der Kontrastsonographie im Überblick und Ausblick in die Zukunft. Radiologe. 2011; 51(8):679.

4 Wermke W, Gaßmann B. Scar Tissue Nodules in Closed Liver Injury. Tumour Diagnostics of the Liver with Echo Enhancers. Berlin, Heidelberg: Springer; 1998.

5 Wermke W. Sonographische Differenzialdiagnose, Leberkrankheiten, Lehrbuch und systematischer Atlas. Deutscher Ärzte-Verlag Köln; 2005.

6 Seitz K, Greis C, Schuler A, Bernatik T, Blank W, Dietrich CF, et al. Frequency of tumor entities among liver tumors of unclear etiology initially detected by sonography in the noncirrhotic or cirrhotic livers of 1349 patients. Results of the DEGUM multicenter study. Ultraschall Med. 2011 Dec;32(6):598-603.

7 Dietrich CF, Potthoff A, Helmberger T, Ignee A, Willmann JK; CEUS LI-RADS Working Group. Standardisierte Befundung und Dokumentation der Kontrastmittelsonografie der Leber (CEUS LI-RADS). Z Gastroenterol. 2018 May;56(5):499-506.

8 Kirstein MM, Vogel A. Epidemiology and Risk Factors of Cholangiocarcinoma. Visc Med. 2016 Dec;32(6):395-400.

9 Hui JY, Yang MK, Cho DH, Li A, Loke TK, Chan JC, et al. Pyogenic liver abscesses caused by Klebsiella pneumoniae: US appearance and aspiration findings. Radiology. 2007 Mar; 242(3):769-76.

10 Kunze G, Staritz M, Köhler M. Contrast-enhanced ultrasound in different stages of pyogenic liver abscess. Ultrasound Med Biol. 2015 Apr;41(4):952-9.

11 Ignee A, Jenssen C, Cui XW, Schuessler G, Dietrich CF. Intracavitary contrast-enhanced ultrasound in abscess drainage-feasibility and clinical value. Scand J Gastroenterol. 2016 Jan;51(1):41-7.
12 Francica G. Intracavitary contrast-enhanced ultrasound in ultrasound-guided percutaneous management of abdominal fluid collections/abscesses by a single clinician: an example of point-of-care ultrasound. J Ultrasound. 2020 Jun;23(2):175-81.

13 Huang DY, Yusuf GT, Daneshi M, Ramnarine R, Deganello A, Sellars ME, et al. Contrast-enhanced ultrasound (CEUS) in abdominal intervention. Abdom Radiol (NY). 2018 Apr;43(4):960-76.

14 Popescu A, Sporea I, Şirli R, Dănilă M, Mare R, Grădinaru Taşcău O, et al. Does Contrast Enhanced Ultrasound improve the management of liver abscesses? A single centre experience. Med Ultrason. 2015 Dec;17(4): 451-5.

15 Luyao Z, Xiaoyan X, Huixiong X, Zuo-Feng X, Guang-Jian L, Ming-de L. Percutaneous ultrasound-guided cholangiography using microbubbles to evaluate the dilated biliary tract: initial experience. Eur Radiol. 2012 Feb; 22(2):371-8.

16 Darge K. Voiding urosonography with ultrasound contrast agents for the diagnosis of vesicoureteric reflux in children. I. Procedure. Pediatr Radiol. 2008 Jan;38(1):40-53. 
17 Heinzmann A, Müller T, Leitlein J, Braun B, Kubicka S, Blank W. Endocavitary contrast enhanced ultrasound (CEUS)-work in progress. Ultraschall Med. 2012 Feb;33(1):76-84.

18 Ignee A, Atkinson NS, Schuessler G, Dietrich CF. Ultrasound contrast agents. Endosc Ultrasound. 2016 Nov-Dec;5(6):355-62.

19 Sidhu PS, Cantisani V, Dietrich CF, Gilja OH, Saftoiu A, Bartels E, et al. The EFSUMB Guidelines and Recommendations for the Clinical Practice of Contrast-Enhanced Ultrasound (CEUS) in Non-Hepatic Applications: Update 2017 (Long Version). Ultraschall Med. 2018 Apr;39(2):e2-44.

20 Sun Y, Yang S, Qi E, Liu F, Zhou F, Lu Y, et al. Comparative Diagnostic Evaluation with Contrast-Enhanced Ultrasound, Computed Tomography and Magnetic Resonance Imaging in Patients with Pancreatic Cystic Neoplasms. Cancer Manag Res. 2020 Apr;12: 2889-98.

21 Hackert T, Fritz S, Klauss M, Bergmann F, Hinz U, Strobel O, et al. Main-duct Intraductal Papillary Mucinous Neoplasm: High Cancer Risk in Duct Diameter of 5 to $9 \mathrm{~mm}$. Ann Surg. 2015 Nov;262(5):875-80.

22 Tanaka M. Thirty years of experience with intraductal papillary mucinous neoplasm of the pancreas: from discovery to international consensus. Digestion. 2014;90(4):265-72.

23 Anand N, Sampath K, Wu BU. Cyst features and risk of malignancy in intraductal papillary mucinous neoplasms of the pancreas: a meta-analysis. Clin Gastroenterol Hepatol. 2013 Aug;11(8):913-21.

24 Dörffel Y, Wermke W. Neuroendocrine tumors: characterization with contrast-enhanced ultrasonography. Ultraschall Med. 2008 Oct;29(5):506-14.

25 Dietrich CF, Jenssen C. Modern ultrasound imaging of pancreatic tumors. Ultrasonography. 2020 Apr;39(2):105-13.

26 Vitali F, Pfeifer L, Janson C, Goertz RS, Neurath MF, Strobel D, et al. Quantitative perfusion analysis in pancreatic contrast enhanced ultrasound (DCE-US): a promising tool for the differentiation between autoimmune pancreatitis and pancreatic cancer. Z Gastroenterol. 2015 Oct;53(10):1175-81.
27 Kunze G, Seitz K, Mauch M, Petersen F. Clinical Ultrasound in Inflammatory Bowel Disease. Ultraschall Med. 2019 Apr;40(2):13262.

28 Mocci G, Migaleddu V, Cabras F, Sirigu D, Scanu D, Virgilio G, et al. SICUS and CEUS imaging in Crohn's disease: an update. J Ultrasound. 2017 Jan;20(1):1-9.

29 Kinkel H, Michels G, Jaspers N. Ultraschall zur Diagnostik und Verlaufskontrolle chronisch-entzündlicher Darmerkrankungen [Value of ultrasound in diagnostic and follow-up of chronic inflammatory bowel diseases]. Dtsch Med Wochenschr. 2015 Jan; 140(1):46-50.

30 Weiss D, van der Weg B, Frenzer A. Prospective Study of Contrast enhanced ultrasound (CEUS) in Patients with Crohn's Disease. Jahreskongress Schweizerische Gesellschaft für Gastroenterologie; 2011.

31 Ignee A, Jenssen C, Hocke M, Dong Y, Wang WP, Cui XW, et al. Contrast-enhanced (endoscopic) ultrasound and endoscopic ultrasound elastography in gastrointestinal stromal tumors. Endosc Ultrasound. 2017 JanFeb;6(1):55-60.

32 Bernatik T, Schuler A, Kunze G, Mauch M, Dietrich CF, Dirks K, et al. Benefit of Contrast-Enhanced Ultrasound (CEUS) in the Follow-Up Care of Patients with Colon Cancer: A Prospective Multicenter Study. Ultraschall Med. 2015 Dec;36(6):590-3.

33 Paprottka PM, Roßpunt S, Ingrisch M, Cyran CC, Nikolaou K, Reiser MF, et al. Reducing tumor growth and angiogenesis using a triple therapy measured with Contrast-enhanced ultrasound (CEUS). BMC Cancer. 2015 May; 15(1):373.

34 Wu Z, Yang X, Chen L, Wang Z, Shi Y, Mao $\mathrm{H}$, et al. Anti-angiogenic therapy with contrast-enhanced ultrasound in colorectal cancer patients with liver metastasis. Medicine (Baltimore). 2017 May;96(20):e6731

35 Amadori M, Barone D, Scarpi E, Oboldi D, Amadori E, Bandi G, et al. Dynamic contrastenhanced ultrasonography (D-CEUS) for the early prediction of bevacizumab efficacy in patients with metastatic colorectal cancer. Eur Radiol. 2018 Jul;28(7):2969-78.
36 Zocco MA, Garcovich M, Lupascu A, Di Stasio E, Roccarina D, Annicchiarico BE, et al. Early prediction of response to sorafenib in patients with advanced hepatocellular carcinoma: the role of dynamic contrast enhanced ultrasound. J Hepatol. 2013 Nov;59(5):1014-21.

37 Ultraschall in der Chirurgie. Simanowski JH, Mendel V, editors. Intraoperative und interventionelle Sonographie. Springer-Verlag GmbH; 1991. pp 205.

38 Simanowski JH. Blunt trauma and acute diseases of the abdomen and chest: free fluid what now? Ultraschall Med. 2019 Oct;40(5): 552-9.

39 Hsiao CY, Chen PD, Huang KW. A Prospective Assessment of the Diagnostic Value of Contrast-Enhanced Ultrasound, Dynamic Computed Tomography and Magnetic Resonance Imaging for Patients with Small Liver Tumors. J Clin Med. 2019 Sep;8(9):1353.

40 Yang D, Li R, Zhang XH, Tang CL, Ma KS, Guo DY, et al. Perfusion Characteristics of Hepatocellular Carcinoma at Contrast-enhanced Ultrasound: Influence of the Cellular differentiation, the Tumor Size and the Underlying Hepatic Condition. Sci Rep. 2018 Mar;8(1):4713.

41 Schellhaas B, Pfeifer L, Kielisch C, Goertz RS, Neurath MF, Strobel D. Interobserver Agreement for Contrast-Enhanced Ultrasound (CEUS)-Based Standardized Algorithms for the Diagnosis of Hepatocellular Carcinoma in High-Risk Patients. Ultraschall Med. 2018 Dec;39(6):667-74.

42 Schwarze V, Marschner C, Völckers W, Grosu S, Negrão de Figueiredo G, Rübenthaler J, et al. Diagnostic value of contrast-enhanced ultrasound versus computed tomography for hepatocellular carcinoma: a retrospective, single-center evaluation of 234 patients. J Int Med Res. 2020 Jun;48(6):300060520930151.

43 Dietrich CF, Burmester E. Contrast-enhanced ultrasound of small focal solid pancreatic lesions: A must! Endosc Ultrasound. 2017 Dec; 6(9 Suppl 3):S106-10

44 Fan Z, Yan K, Wang Y, Qiu J, Wu W, Yang L, et al. Application of Contrast-Enhanced Ultrasound in Cystic Pancreatic Lesions Using a Simplified Classification Diagnostic Criterion. BioMed Res Int. 2015;2015:974621. 